\title{
Metodologia TECLIM para uso racional de água na indústria: o banco de ideias no contexto da metodologia front-end loading
}

\author{
TECLIM method for rational water use in industry: \\ the bank of ideas in the context of the front-end loading methodology \\ Geiza Lima de Oliveira', Asher Kiperstok², Ricardo de Araújo Kalid³, \\ Karla Patrícia Santos Oliveira Rodríguez Esquerre ${ }^{4}$, Emerson Andrade Sales ${ }^{5}$
}

口-

\begin{abstract}
RESUMO
O banco de ideias (BI) é uma ferramenta da metodologia TECLIM para otimização ambiental de processos industriais. Está conceitualmente associado com a metodologia front-end loading (FEL), a qual considera que forte atenção nas etapas iniciais de identificação e seleção de alternativas aumenta as chances de sucesso de um projeto, evitando que recursos sejam desperdiçados ou mal alocados. O BI abrange a etapa FEL 1, na qual é feito o levantamento de ideias, e segue até parte da etapa FEL 2, na qual as oportunidades selecionadas são desenvolvidas a projetos conceituais. O método para priorização de oportunidades no Bl é apresentado demonstrando-se a sinergia com a FEL. A aplicação do BI permitiu o cadastro e processamento de 500 ideias com potencial de redução do consumo de água em $1,4 \times 10^{3}$ t.h $h^{-1}$ e de efluentes em pelo menos $0,5 \times 10^{3}$ th.h'. O BI direciona recursos para projetos conceituais mais atrativos e sustentáveis.
\end{abstract}

Palavras-chave: banco de ideias TECLIM; metodologia FEL; uso de água na indústria; produção mais limpa; uso racional de água.

\begin{abstract}
The bank of ideas (BI) is a TECLIM tool for environmental optimization of industrial processes. It is conceptually associated with the front-end loading (FEL) methodology, which pays particular attention to the initial stage of identification and selection of alternatives of water use, increasing the chances of a project's success, avoiding wasted or badly allocated resources. The BI covers FEL 1 stage, in which the ideas are invited, followed by stage FEL 2, in which the opportunities selected are developed into conceptual projects. The method of giving priorities to the opportunities in the $\mathrm{BI}$ is presented, showing the synergy with FEL. The application of the $\mathrm{Bl}$ enabled the register and process of 500 ideas with the potential to reduce water consumption by $1.4 \times 10^{3}$ t.h-1 and effluents by at least $0.5 \times 10^{3}$ t.h $^{-1}$. The $\mathrm{BI}$ tool helps to identify the most attractive and sustainable conceptual projects.
\end{abstract}

Keywords: TECLIM ideas bank; front-end loading; use of water in industry; cleaner production; rational use of water

\section{INTRODUÇÃO}

A água é apontada como um dos fatores ambientais críticos para as próximas duas décadas (UNEP, 2011). O estresse hídrico, provocado pela retirada excessiva das águas de superfície e dos aquíferos subterrâneos e pela poluição e uso ineficiente dos recursos de água doce (ANA \& CEBDS, 2006), aliado à interferência das mudanças climáticas na disponibilidade desse recurso devem estimular ainda mais a busca de soluções de curto a médio prazo para atendimento aos diversos usos da água das populações atuais e futuras.

Dentre os usos consuntivos da água, estima-se que o setor industrial seja responsável por cerca de $25 \%$ do consumo de água no mundo (METCALF \& EDDY, 2003) e 18\% do consumo de água no Brasil (ANA, 2009). A restrição da água na indústria pode ocasionar a perda da licença para operar, aumento do custo de

\footnotetext{
'Mestre em Engenharia Industrial pela Universidade Federal da Bahia (UFBA). Engenheira de Meio Ambiente na Cristal Pigmentos do Brasil - Camaçari (BA), Brasil. ${ }^{2}$ PhD em Engenharia Química, Tecnologias Ambientais pela University Of Manchester Institute of Science and Technology. Professor do Programa de Engenharia Industrial da UFBA - Salvador (BA), Brasil.

${ }^{3}$ Doutor em Engenharia Química pela Universidade de São Paulo (USP). Professor da Universidade Federal do Sul da Bahia (UFSB) - Itabuna (BA), Brasil.

${ }^{4}$ Doutora em Engenharia Química pela Estadual de Campinas (UNICAMP). Professora do Programa de Engenharia Industrial da UFBA - Salvador (BA), Brasil.

${ }^{5}$ Doutor em Ciências/Engenharia Química pela Université Paris VII - Denis Diderot. Professor Associado da UFBA - Salvador (BA), Brasil.

Endereço para correspondência: Geiza Lima de Oliveira - Escola Politécnica da Universidade Federal da Bahia, TECLIM, DEA, 4 O andar - Rua Aristides Novis, O2 -

Federação - 40210-630 - Salvador (BA), Brasil - E-mail: engeuqui@gmail.com

Recebido: 13/01/13 - Aceito: 21/03/16 - Reg. ABES: 109240
} 
produção, além do aumento da pressão regulamentar e das comunidades (WBCSD, 2010).

Para que as indústrias minimizem os problemas relativos à água é recomendada a formação de parcerias com municipalidades para desenvolver opções mais baratas de fornecimento de água e saneamento, com grupos não governamentais para encorajar a melhoria e preservação dos recursos hídricos e com a comunidade científica onde atua, para a conscientização quanto ao uso e gestão dos recursos hídricos e desenvolvimento de tecnologias que melhor aproveitem o ciclo da água, além da adoção de programas para uso racional desse recurso (OLIVER; RODRÍGUEZ; UDAQUIOLA, 2008; ANA \& CEBDS, 2006).

$\mathrm{Na}$ Bahia, a formação de parceria com a universidade tem sido adotada por indústrias como forma de identificação de oportunidades para redução do consumo da água e da geração de efluentes. Com base nessa experiência, a Rede de Tecnologias Limpas (TECLIM) da Universidade Federal da Bahia (UFBA) desenvolveu uma metodologia específica para uso racional da água na indústria baseada na inserção, adaptação ou criação de novas ferramentas de produção mais limpa $(\mathrm{P}+\mathrm{L})$ nos diversos níveis hierárquicos da empresa.

O objetivo da metodologia TECLIM é provocar a mudança de percepção quanto ao uso da água e motivar o surgimento de ideias e geração de oportunidades para a empresa. Essa metodologia encontra-se parcialmente definida em Kiperstok, Kalid e Sales (2006) e Kiperstok et al. (2011) e consiste atualmente de 16 ferramentas:

1. montagem do ecotime, com pessoas comprometidas, com formação multidisciplinar e com experiências e habilidades complementares;

2. construção de uma parceria entre universidade e indústria;

3. inserção dos conceitos de $\mathrm{P}+\mathrm{L}$ através da capacitação permanente e em larga escala;

4. utilização de ferramentas de acompanhamento de projetos: MS-Project, curva S, reuniões semanais de toda a equipe, reuniões quadrimestrais com a direção da empresa (Project Management Office - PMO);

5. medição e conhecimento das vazões das correntes através de balanço hídrico com reconciliação de dados, que considera a qualidade da informação de cada fonte de dados (MARTINS et al., 2010);

6. desenvolvimento de modelos fenomenológicos ou empíricos (MENEZES; OLIVEIRA-ESQUERRE; KALID, 2011) para operações unitárias ou processos de tratamento, movimentação ou armazenamento de água;

7. implementação de um banco de ideias (BI) que considera aspectos culturais, ambientais e econômicos na avaliação do potencial ou dificuldade para execução de uma oportunidade;

8. implantação de um sistema de informações geográficas (SIG) georreferenciando as fontes produtoras e consumidoras de água dentro da empresa e no seu contexto regional (CARVALHO et al., 2009);
9. otimização a partir da aplicação de conceitos e instrumentos para a síntese de redes de transferência de massa (GOMES; QUEIROZ; PESSOA, 2007);

10. análise da inserção da empresa no ciclo hidrológico regional (OLIVEIRA-ESQUERRE et al., 2009; 2010);

11. elaboração de projetos conceituais de minimização do uso da água e geração de efluentes;

12. auditoria de fontes de efluentes (ÁVILA, 1995);

13. análise de desvios ambientais;

14. cálculo de indicadores ambientais e sua incerteza (MENDES et al., 2011) a partir de balanço de massa reconciliado;

15. acompanhamento e monitoramento do consumo de água através do sistema AGUAPURA;

16. avaliação dos resultados e mudanças culturais que ocorreram após o término do projeto cooperativo.

Este trabalho apresenta a etapa referente à implementação de um BI, ferramenta 7, que considera aspectos culturais, ambientais e econômicos na avaliação do potencial ou dificuldade para execução de uma oportunidade, abrangendo também a elaboração de projetos conceituais, ferramenta 11.

$\mathrm{O}$ BI está conceitualmente associado à metodologia front-end loading (FEL), que foca na potencialização das fases iniciais do projeto como forma de escolher as oportunidades mais promissoras para a empresa, resultando em ganhos de orçamento e prazo de execução, diminuição dos riscos, redução dos custos e, consequentemente, otimização do retorno do investimento (RUTKOWSKI, 2010).

Pela revisão da literatura, os princípios da metodologia FEL são amplamente aplicados na indústria de petróleo e gás (JERGEAS, 2008; SPANGLER 2005), empresas de manufatura (MORITA; FLYNN; OCHIAI, 2011), na captura e estocagem de gás carbônico (KERKHOF \& VAN BIRGELEN, 2011; ARAYA; CULLICK; SMYTH, 2011) e na indústria da construção (JERGEAS, 2009). Em relação ao uso da metodologia FEL para gestão de projetos relacionados ao uso da água pelas plantas industriais, não foram encontradas publicações abordando esse tema.

No Brasil, a metodologia FEL está sendo cada vez mais utilizada pelas grandes empresas (LUCCI; CONCER; SANTANA, 2009). Entretanto, no meio acadêmico tem sido objeto de poucos estudos, configurando uma oportunidade de pesquisa (MORAES, 2010).

A ferramenta BI da metodologia TECLIM será então apresentada no contexto FEL. A sinergia entre ambas as ferramentas será delineada. Os resultados obtidos nos projetos da TECLIM em termos de potenciais estimados de redução do consumo de água e da geração de efluentes; percentual de ideias priorizadas e dificuldades encontradas para levantamento e desenvolvimento das ideias, oportunidades e projetos conceituais também são apresentados. 
O objetivo é demonstrar a eficiência dos conceitos propostos pela FEL empregando uma metodologia amplamente utilizada na tomada de decisão em projetos de grandes empreendimentos para a realidade da gestão do uso racional da água em uma indústria.

Ao final, pretende-se mostrar a eficiência da ferramenta BI para apoio à decisão dos gestores do uso da água pela empresa e a possibilidade de expansão de seu uso para outros setores industriais e urbanos, ou com relação a outros recursos naturais, os quais também constituem linhas de pesquisa da Rede TECLIM (www.TECLIM.ufba.br).

\section{METODOLOGIA FRONT-END LOADING}

A metodologia FEL para desenvolvimento de projetos foi desenvolvida pelo Independent Project Analysis (IPA) e é aplicada no pré-planejamento de projetos de megaempreendimentos denominados projetos de capital. O IPA propõe a FEL como forma de aumentar a produtividade capital do projeto, evitando que investimentos sejam feitos em projetos que ao longo do seu ciclo de vida sejam considerados desvantajosos ou inviáveis (IPA, 2011; RUTKOWSKI, 2010; LUCCI; CONCER; SANTANA, 2009). A potencialização das etapas iniciais minimiza as mudanças durante a fase de execução de projetos, onde os custos para efetuar tais mudanças são mais elevados (BARSHOP, 2004).

As fases de iniciação e planejamento de um projeto (PMI, 2004) possuem um nível de incertezas elevado, devido às informações estarem mais difusas ou mesmo serem inexistentes. A qualidade e a consistência das etapas iniciais do projeto predizem os principais resultados e impactam diretamente no sucesso do projeto, pois nelas são definidos escopo, prazo, custo, recursos e efetuado o levantamento dos riscos do projeto (MERROW et al., 2009; SANTIAGO et al., 2008). A metodologia FEL consiste, portanto, em um processo para o desenvolvimento de projetos competitivos que se baseiam na medição do desempenho e aumento do nível de definição do empreendimento com forte atenção às atividades iniciais de um projeto; sendo a avaliação econômica o principal parâmetro para estabelecimento da métrica de desempenho de um projeto (SAPUTELLI et al., 2008).

Além das análises econômicas e técnicas, a FEL prevê também avaliação de saúde, segurança, meio ambiente e relacionamento com as comunidades, em todas as etapas (VALE, 2007). Ou seja, a metodologia FEL, quando executada adequadamente, proporciona menor custo total, aliada ao atendimento das metas de qualidade, segurança, meio ambiente e do escopo do empreendimento (BATAVIA, 2001).

Entretanto, o uso dessa metodologia apresenta limitações devido à terminologia na área de gestão de projetos não ser uniforme, podendo existir trabalhos abordando os princípios da FEL com outros termos (WEIDJE, 2008), com destaque para front-end development, front-end design (FED), front-end engineering desing (FEED) e pre-project planning (PPP). Neste trabalho, a definição adotada é a do FEL proposta pelo Independent Project Analysis (IPA). Geralmente a FEL é desenvolvida em três etapas distintas, embora alguns trabalhos abordem quatro (LUCCI; CONCER; SANTANA, 2009) e até oito etapas (RUTKOWSKI, 2010). Neste trabalho, adotou-se as três etapas conforme definidas pela IPA como: FEL 1 (planejamento de negócios), FEL 2 (desenvolvimento de escopo) e FEL 3 (planejamento do projeto).

A etapa FEL 1 tem por objetivo definir a oportunidade de negócio. Nessa etapa são levantadas todas as possíveis opções para desenvolvimento do projeto, sendo selecionadas as que seguirão para desenvolvimento da etapa de FEL 2 (MORAES, 2010). Após um possível projeto ter sido identificado, ele deve passar por certa quantidade de detalhamento, que pode incluir uma avaliação técnica, o desenvolvimento de um cronograma de marcos, e uma estimativa inicial de custos (IPA, 2011)

Na etapa FEL 2 é selecionada a oportunidade e desenvolvido o projeto conceitual. São levantadas as informações que atendam à definição do escopo, bem como os critérios e restrições para o desenvolvimento do projeto conceitual (IPA, 2011). O objetivo é comparar as opções e definir, através do resultado da avaliação econômico-financeira, as alternativas que apresentem melhores indicadores econômicos, tais como valor presente líquido (VPL) menor que zero, desprezando-se (ou arquivando) as demais opções (MORAES, 2010).

A FEL 3 é definida como o ponto em que uma das alternativas avaliadas durante a FEL 2 foi selecionada para melhor definição. Nessa fase é elaborado o projeto básico para autorização do financiamento e implantação do projeto. O objetivo do FEL 3 é desenvolver um conjunto de documentos de engenharia (pacote base do projeto) que incorporem condições específicas do local e um plano para executar o projeto. $\mathrm{O}$ fim da etapa FEL 3 ocorre quando a implantação do projeto é autorizada ou abandonada devido a mudanças nas premissas do projeto (IPA, 2011).

De forma resumida, na FEL 1 é desenvolvida e avaliada a oportunidade de investimento e atratividade do negócio, na FEL 2 é feito o estudo e a seleção das alternativas que serão desenvolvidas na FEL 3 e, por sua vez, na FEL 3 é desenvolvido o projeto básico e o planejamento da fase de execução (MORAES, 2010).

Dentre as etapas da metodologia FEL existem portões de aprovação para a continuidade ou não na etapa seguinte, conforme Figura 1.

Para cada etapa FEL, um pacote de documentos deve ser preparado e entregue, e uma avaliação econômica deve ser feita para justificar a passagem para a próxima etapa (SPANGLER, 2005). A cada portão é tomada a decisão se a ideia avança para a fase seguinte, é descartada ou arquivada, ou retorna para ser mais bem definida ou compreendida. Quando um projeto avança através dos estágios, o rigor desse processo aumenta, correspondendo ao aumento dos recursos e esforços requeridos para completar entregas dos estágios. Conforme Figura 1, o percentual de ideias abandonados entre os portões é de $75 \%$ para a o portão 1 , $50 \%$ das restantes para o portão 2 e $1 \%$ das restantes para o portão 3 . 
Para mensurar o grau de definição do projeto, a IPA utiliza índices como o FEL Index, composto de três fatores igualmente importantes: a integralidade do trabalho em um conjunto de itens específicos, o status do projeto de engenharia para instalação e o status do planejamento de execução do projeto. Cada fator contém atividades específicas que devem ser atendidas nas fases FEL 1, FEL 2 e FEL 3 (BARSHOP, 2004; JOSHI, 2003; MERROW, 2009).

Para cada fator avaliado no FEL Index é atribuída uma nota de 1 a 4, representando o nível com que essas atividades foram desenvolvidas. A nota final, dada pela soma das notas atribuídas a cada fator, qualifica o projeto como deficiente (entre 7,5 e 12,0), pobre (entre 6,5 e 7,5), regular (entre 5,5 e 6,0), bom (entre 4,5 e 5,5) e melhores práticas (entre 3,0 e 4,5). Quanto menor o índice FEL, maior a maturidade do projeto, o que para a IPA corresponde à maior probabilidade de sucesso na implementação, ao contrário dos projetos que possuem índice FEL elevado, que deverão ter seus custos aumentados durante a fase de execução (MORAES, 2010).

\section{Banco de ideias: mais do que um brainstorm eletrônico pela internet}

Desde o nascedouro, o BI, sétima ferramenta da metodologia TECLIM para uso racional da água, foi concebido para ser um repositório de ideias, acessível de qualquer lugar ou momento pela internet, e um ambiente para processamento e discussão das ideias. O objetivo é permitir o cadastro livre de ideias que contribuam para a redução do consumo de água e geração de efluentes na indústria. Contudo, o BI é também um sistema para gestão das ideais e priorização das mesmas a oportunidades e em seguida a projetos conceituais, décima primeira ferramenta da metodologia.

Devido à sua aplicação na seleção de alternativas, o BI está conceitualmente associado à etapa FEL 1 (concepção). Os projetos TECLIM se estendem até parte da FEL 2, quando é realizada a seleção de alternativas e o desenvolvimento de projetos conceituais, conforme será apresentado.

Os integrantes dos projetos do TECLIM com a indústria, tanto por parte da universidade quanto por parte da empresa, são orientados e incentivados a registrarem suas ideias no BI, o qual é disponibilizado no sítio www.teclim.ufba.br/ideia, acessado por meio de usuário e senha específicos para cada projeto, visando garantir o sigilo das informações.

O cadastro de ideias no BI possui um formulário obrigatório e simplificado; e outro formulário complementar e mais detalhado (OLIVEIRA et al., 2009). Como itens obrigatórios estão: categoria da ideia (água, efluente, energia elétrica); nome, telefone e e-mail do proponente para contato; título da ideia; definição da área/unidade/equipamento a que esta se refere; o momento em que a ideia deve ser aplicada no processo (exemplo: durante uso indevido de água de incêndio) e uma descrição de ações e resultados previstos com a implementação da ideia. Preenchendo esses campos e efetuando o salvamento, o BI gera o número da ideia com a respectiva data de inserção. O formulário obrigatório é preenchido

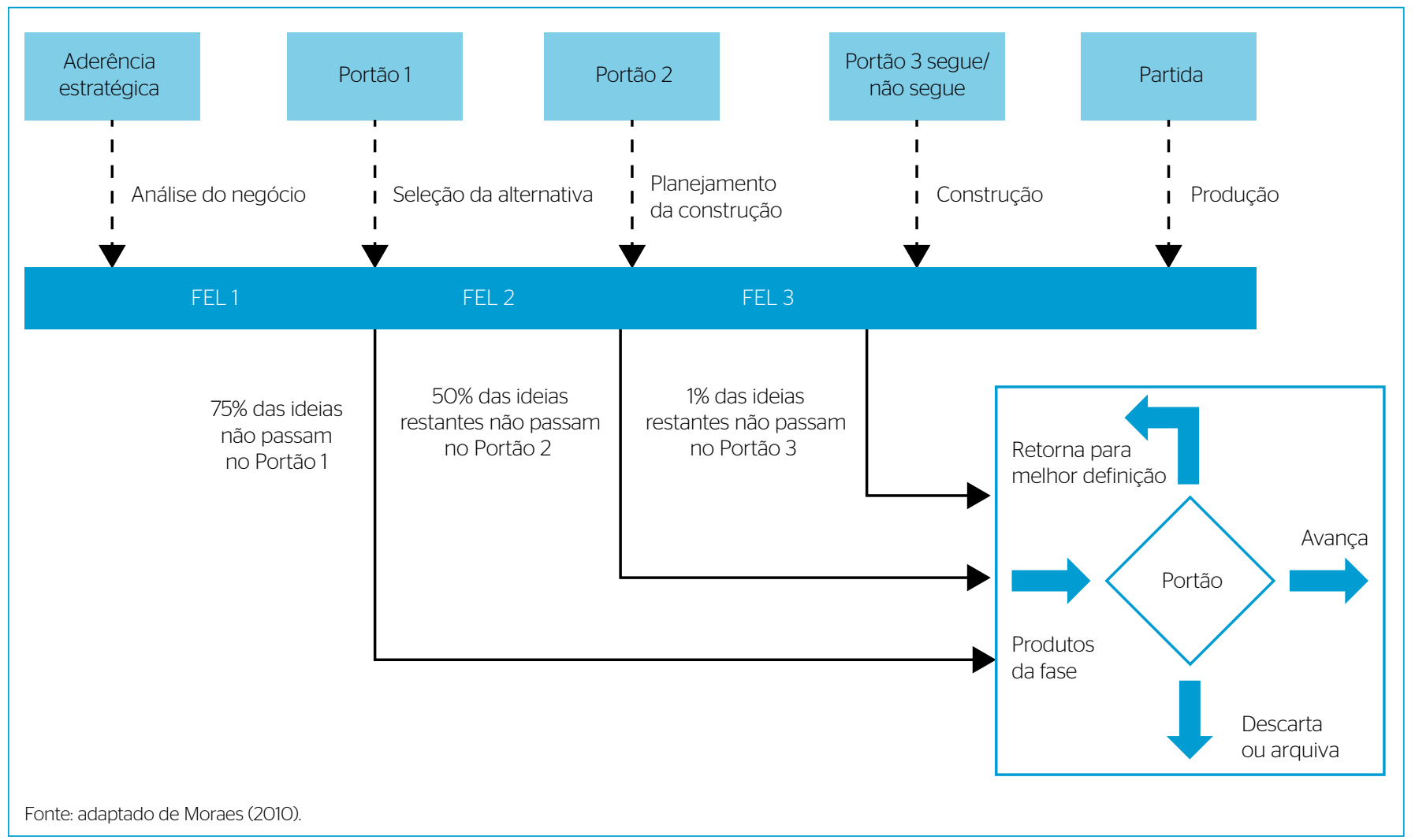

Figura 1 - Processo de validação nos portões. 
pelo autor da ideia, que não precisa ser rigoroso no preenchimento do mesmo, pois o importante é registrar a ideia aproveitando a inspiração.

O formulário complementar é composto por observações gerais; escolha do impacto e do investimento financeiro associado à ideia (a ser apresentado em seguida); resultados que podem ser obtidos com a ideia incluindo uma estimativa de ganho econômico; possíveis barreiras e dificuldades que podem interferir na implementação da ideia, e ações que estejam sendo executadas para desenvolvimento da mesma. $\mathrm{O}$ formulário complementar é preenchido pela equipe do projeto cooperativo encarregado de acompanhar e processar o BI, que precisa ser cuidadoso no preenchimento do mesmo, pois é o momento de aprimorar a ideia.

As ideias cadastradas no BI são avaliadas pela equipe de pesquisadores da TECLIM e pelos engenheiros da empresa. Em um primeiro momento as ideias são lidas e detalhadas. Caso seja necessário, são feitas consultas ao autor da ideia ou mesmo a especialistas para que as informações estejam as mais claras e completas possíveis.

Em seguida, as ideias são apresentadas em reuniões com a área envolvida, onde os aspectos de investimento e impacto positivo são associados e validados. Por investimento entendem-se os recursos econômicos necessários para a implantação da oportunidade.

O impacto positivo de cada ideia ou oportunidade é analisado sob três dimensões:

- dimensão ambiental: referente à contribuição da implementação da ideia, por exemplo, na redução do consumo de água ou na geração de efluente;

- dimensão cultural e psicossocial: está relacionada às mudanças de atitude e comportamento das pessoas que podem ocorrer dentro e fora da empresa;

- dimensão econômico-financeira: se refere aos valores estimados de retorno financeiro proporcionado pela implementação da oportunidade.

A avaliação dos impactos positivos e do investimento é feita por meio da atribuição de notas. Essas notas (1 a 5) correspondem aos critérios de faixa de valores apresentados na Tabela 1. As faixas de valores devem ser adaptadas ao porte e aos hábitos da empresa, por exemplo, a unidade monetária pode ser em dólares.

Para cada dimensão do impacto positivo é atribuído um peso. Após a avaliação das três dimensões (ambiental, mudança de cultura e retorno financeiro) e atribuição das notas, chega-se à nota para o impacto positivo. Os valores de investimento, conforme a terceira coluna da Tabela 1, e impacto positivo, conforme a quarta, quinta e sexta colunas da Tabela 1.

A partir da caracterização da ideia quanto ao impacto positivo e ao investimento conforme descrito é construído um gráfico conforme Figura 2, que representa a matriz de prioridade das ideias para a empresa.

Em uma análise visual, as ideias localizadas no quadrante superior esquerdo seriam aquelas cuja implementação geraria os maiores impactos positivos com os menores investimentos, consideradas muito atrativas para a empresa. No quadrante esquerdo inferior, encontram-se as ideias com baixo impacto positivo, porém, por exigirem baixos investimentos ainda são consideradas atrativas. No quadrante direito superior estão as ideias com alto impacto positivo, porém necessitam de alto investimento, logo são consideradas pouco atrativas e, por fim, no quadrante direito inferior aparecem as ideias consideradas sem atratividade, pois apresentam baixo impacto positivo e alto investimento. Nas interseções é apresentado o número das ideias correspondente a cada classificação (investimento versus impacto positivo).

O site do BI gera automaticamente a Figura 2 a partir das ideias registradas, detalhadas e avaliadas. Essa matriz é um embasamento inicial para discussão junto à empresa. É possível que nessa discussão e priorização sejam consideradas ideias que em um primeiro momento não apareceram tão atrativas e vice-versa.

Todas as ideias cadastradas após classificadas quanto ao maior impacto positivo e investimento são hierarquizadas em uma oficina na qual participam engenheiros e técnicos operacionais de vários setores da empresa, além dos professores-orientadores e pesquisadores do projeto. As ideias com prioridades mais alta são renomeadas de oportunidades e partem para um novo ciclo de detalhamento ou

Tabela 1 - Informações base para avaliação da atratividade.

\begin{tabular}{|c|c|c|c|c|c|c|c|c|}
\hline \multirow{2}{*}{ Nota } & \multirow{2}{*}{ Conceito } & \multirow{2}{*}{ Investimento inicial + operacional (R\$. ano-1) } & \multicolumn{6}{|c|}{ Impacto positivo } \\
\hline & & & Peso & 5 & Peso & 2 & Peso & 3 \\
\hline 2 & Baixo & $5.000,01$ a $50.000,00$ & \multicolumn{2}{|c|}{0,1 a 0,4} & \multicolumn{2}{|c|}{ Área } & \multicolumn{2}{|c|}{$\begin{array}{l}5.000,01 \mathrm{a} \\
50.000,00\end{array}$} \\
\hline 4 & Alto & 500.000,01 a 1.000.000,00 & \multicolumn{2}{|c|}{2,1 a 4,0} & \multicolumn{2}{|c|}{$\begin{array}{l}\text { Planta + residência } \\
\text { do funcionário }\end{array}$} & \multicolumn{2}{|c|}{$\begin{array}{l}500.000,01 \mathrm{a} \\
1.000 .000,00\end{array}$} \\
\hline 5 & Muito alto & Mais do que 1.000.000,01 & \multicolumn{2}{|c|}{ Acima de 4,0} & \multicolumn{2}{|c|}{$\begin{array}{c}\text { Planta + residência } \\
\text { do funcionário + } \\
\text { comunidade }\end{array}$} & \multicolumn{2}{|c|}{$\begin{array}{l}\text { Mais do que } \\
1.000 .000,01\end{array}$} \\
\hline
\end{tabular}




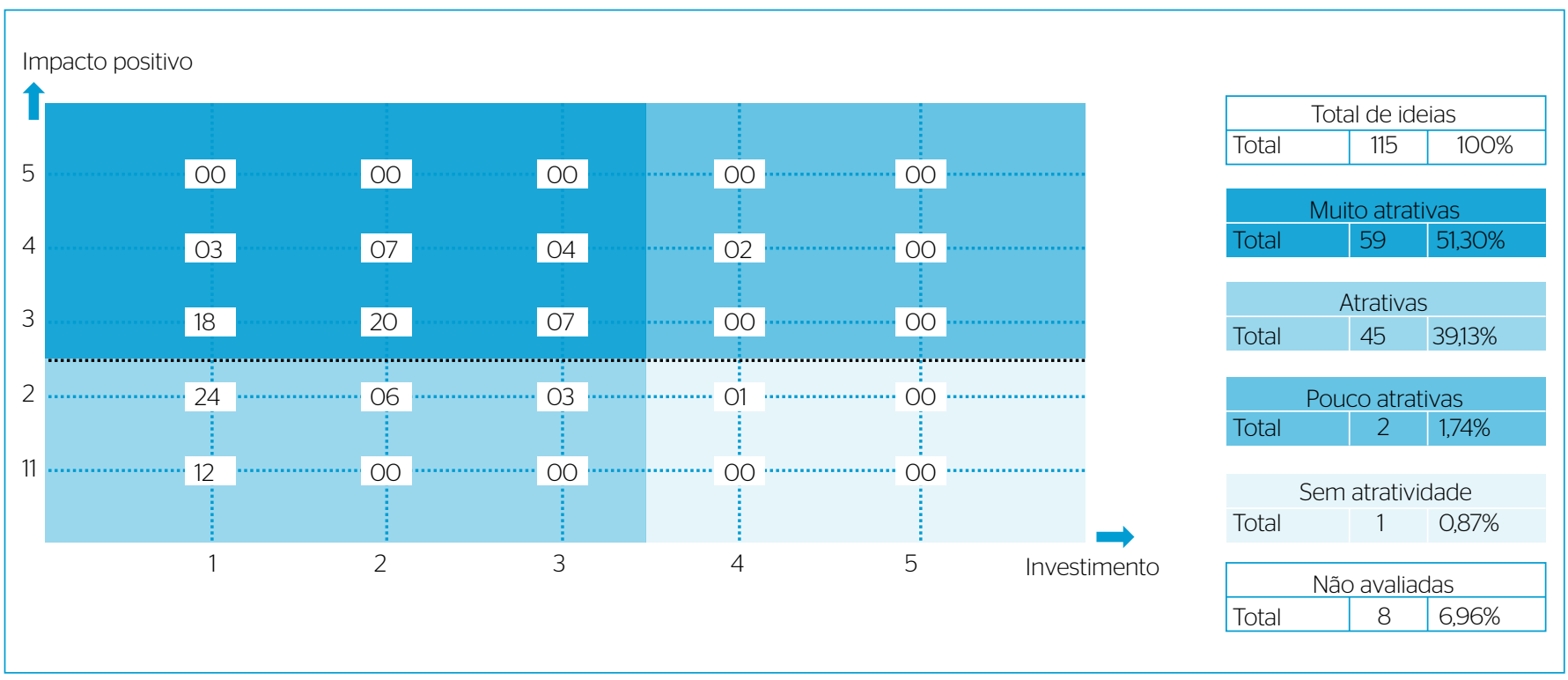

Figura 2 - Exemplo de uma matriz de prioridade das ideias (dados referentes ao projeto E).

aprofundamento. As oportunidades são então desenvolvidas e depois hierarquizadas numa segunda oficina com os mesmos participantes da oficina de ideias onde são selecionadas as oportunidades que serão desenvolvidas até a categoria de "projetos conceituais".

Esses ciclos de oficina de ideias, oficina de oportunidades e desenvolvimento de projetos conceituais se repetem continuamente até o final do projeto, sendo que alguns desses ciclos ficam inconclusos, à espera da prorrogação ou renovação do projeto cooperativo conforme mostrado na Figura 3.

Esse procedimento cíclico tem o intuito de concentrar os recursos disponíveis nas ideias, oportunidades e projetos conceituais mais promissores, evitando o desperdício de tempo e a não avaliação de uma alternativa mais atrativa.

Porém, a metodologia prevê que a qualquer instante, antes, durante ou depois de uma oficina, uma ideia, oportunidade ou projeto conceitual pode ser sacado e elegido como uma alternativa a ser encaminhada para o portfólio de projetos da empresa, ou seja, uma ideia, por exemplo, pode pular etapas e ir diretamente para o FEL 3, a depender da decisão da empresa. Isso de fato ocorre, pois certas circunstâncias podem favorecer a realização de um investimento, por exemplo, uma súbita disponibilidade de recursos financeiros para realizar projetos ambientalmente impactantes ou por interesse estratégicos da empresa em realizar investimentos ambientalmente mais significativos.

Um projeto conceitual pode reunir mais de uma ideia que sejam consideradas semelhantes ou complementares; essas ideias são compiladas em uma ideia resumo.

A entrega de projetos conceituais não significa que a empresa perca as demais oportunidades cadastradas no BI. Os projetos conceituais representam as oportunidades mais passíveis de implantação. Seu desenvolvimento e execução, entretanto, ficam a critério da empresa.

A retenção de ideias nos portões de aprovação característica da metodologia FEL, mostrada na Figura 1, também é observada no BI, conforme será mostrado nos resultados. Como o prazo dos projetos é inicialmente curto (de um a dois anos) e a disponibilidade de tempo para priorização de ideias é reduzida, é importante priorizar esforços para que as alternativas selecionadas sejam as melhores e estejam alinhadas com os princípios da $\mathrm{P}+\mathrm{L}$ e com a estratégia da empresa.

As obrigações dos projetos cooperativos encerram-se com a entrega dos projetos conceituais. Contudo a TECLIM continua prestando assistência, sempre que requisitada, com relação às ideias, oportunidades ou projetos conceituais registrados no BI.

Para que o BI seja amplamente utilizado no projeto, sua divulgação é contemplada e seu uso reforçado continuamente. Normalmente o BI é também apresentado nos treinamentos (terceira ferramenta da metodologia) e divulgado em seminários, reuniões e oficinas com a participação de pesquisadores da TECLIM e operadores, técnicos e engenheiros da empresa parceira.

O acesso à ferramenta continuará disponível após o encerramento do projeto com a UFBA. O ideal é que a empresa continue alimentando o BI e volte a fazer rodadas de priorização sem descartar oportunidades mais antigas. Como foi dito anteriormente, a mudança de cenários da empresa pode fazê-la recuperar uma oportunidade que tenha sido anteriormente descartada ou rebaixar uma oportunidade inicialmente considerada prioritária.

A metodologia TECLIM desenvolvida nos projetos com a indústria foi adaptada e expandida para outros projetos da TECLIM em 
áreas urbanas e domiciliares. O BI foi aplicado, por exemplo, no projeto de racionalização de água no Aeroporto Internacional de Salvador, Bahia (KIPERSTOK; FREIRE; KALID, 2010) e atualmente está sendo utilizado no projeto de uso racional de água em residências e prédios públicos e em um shopping center de Salvador. O uso da ferramenta por empresas, instituições ou pessoas é possível bastando o interessado cadastrar-se através do sítio www.teclim.ufba.br.

\section{Sinergia entre o banco de ideias}

\section{teclim e a metodologia front-end loading}

Embora os projetos da TECLIM não se tratem de projetos de capital e possuam um ciclo de vida inicialmente curto (de um a dois anos, podendo ser renovados), o BI guarda semelhanças com a metodologia FEL à medida que propõe atingir o maior detalhamento possível nas etapas iniciais de seleção de alternativas e escolha de oportunidade para otimização ambiental da empresa em estudo, antes de definir quais as oportunidades mais promissoras para essa otimização.

O levantamento de ideias e o desenvolvimento das mesmas até oportunidades priorizadas é feito desde o início do projeto cooperativo, na fase de concepção FEL 1, com a identificação e avaliação de alternativas, e se estendem até parte da fase conceitual FEL 2, na qual as alternativas são selecionadas e são elaborados os projetos conceituais.

O detalhamento das ideias pela equipe da TECLIM é feito com auxílio dos operadores e engenheiros da indústria em estudo que, por sua vez, dispõem de tempo limitado para essa avaliação em função das suas demais atividades tanto na indústria como no próprio projeto. Por isso

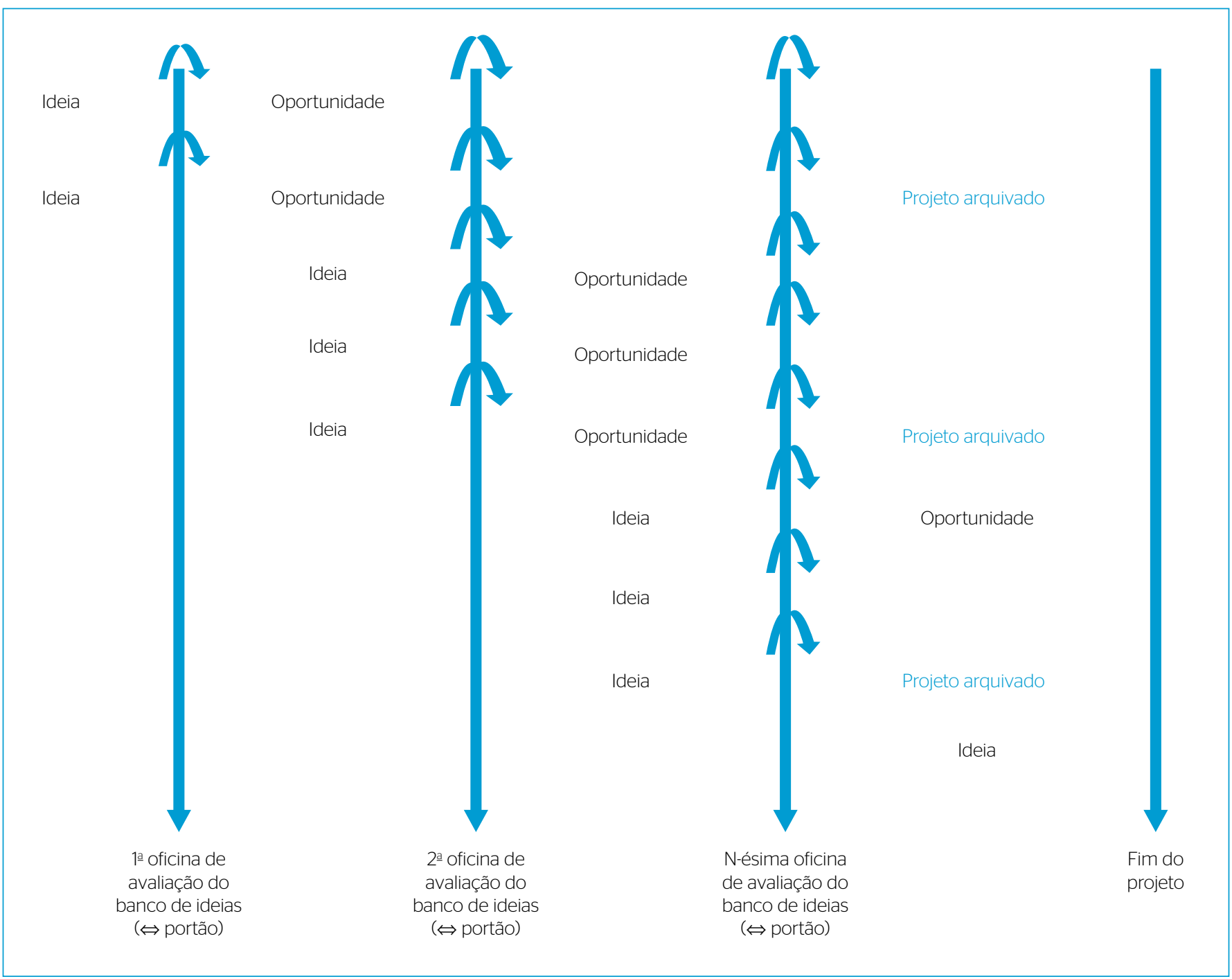

Figura 3 - Rodadas de validação do banco de ideias. Oficinas de hierarquização das ideias, oportunidades e projetos conceituais concebidos. Em destaque uma ideia que pulou etapas e passou diretamente para a categoria de projeto em execução. 
a importância da priorização de esforços para a seleção das oportunidades mais alinhadas com a estratégia da empresa, que terão mais chances de compor seu portfólio de projetos e serem implementados.

A Figura 4 apresenta a sinergia entre o BI e o processo FEL. Assim como no FEL, no BI existem atividades de detalhamento em cada etapa (ideia, oportunidade, projeto conceitual), que correspondem ao grau de definição em cada etapa.

As oficinas da Figura 4 correspondem aos portões de aprovação. No caso do BI existem dois portões na etapa correspondente a FEL 1, o primeiro quando as ideias são elevadas à categoria de oportunidades e o segundo ocorre quando a empresa define quais oportunidades irão gerar os projetos conceituais. Ideia, oportunidade e projeto conceitual vão adquirindo percentual de avanço de 0 a $100 \%$ à medida que os requisitos de detalhamento vão sendo cumpridos.

Parte dos produtos característicos da fase FEL 2, tais como classificação dos riscos e projeto preliminar de equipamentos ficam por conta da empresa, com assessoria da TECLIM quando necessário.

A fase FEL 3, correspondente à implantação e operação do empreendimento, é executada pelas empresas através de recursos próprios ou da contratação de empresas de engenharia, não estando contemplada na metodologia, sendo possível a consultoria à TECLIM nessa fase.

\section{RESULTADOS}

A ferramenta BI foi desenvolvida e aplicada em projetos com a indústria e vem sendo aperfeiçoada nos projetos em andamento pela Rede TECLIM. A quantidade de ideias cadastradas, a duração e o potencial de economia de água e redução de efluentes, estimado nos diversos projetos desenvolvidos pela TECLIM são apresentadas na Tabela 2.

Ao longo dos projetos cooperativos listados na Tabela 2, cerca de 500 ideias foram inseridas nos $\mathrm{BI}$ desses projetos, representando potenciais estimados de economia de água em $1,4 \times 10^{3}$ t.h ${ }^{-1}$ e de redução de efluentes em pelo menos $0,5 \times 10^{3}$ t.h ${ }^{-1}$.

O resultado da avaliação da atratividade das ideias ou oportunidades para a empresa em relação ao investimento e impacto positivo é apresentado na Figura 5. As ideias que não foram discutidas durante o período do projeto aparecem como não avaliadas.

A Figura 5 aponta que a soma dos percentuais de ideias muito atrativas ou atrativas é superior a $70 \%$ em todos os projetos realizados. Na empresa D, essa proporção chega a $98 \%$ do total de ideias cadastradas. Verifica-se, portanto, que a grande maioria das oportunidades identificadas nos projetos de parceria estão alinhadas com a estratégia da empresa e podem ser implantadas com baixo investimento e resultarão em um alto impacto positivo.

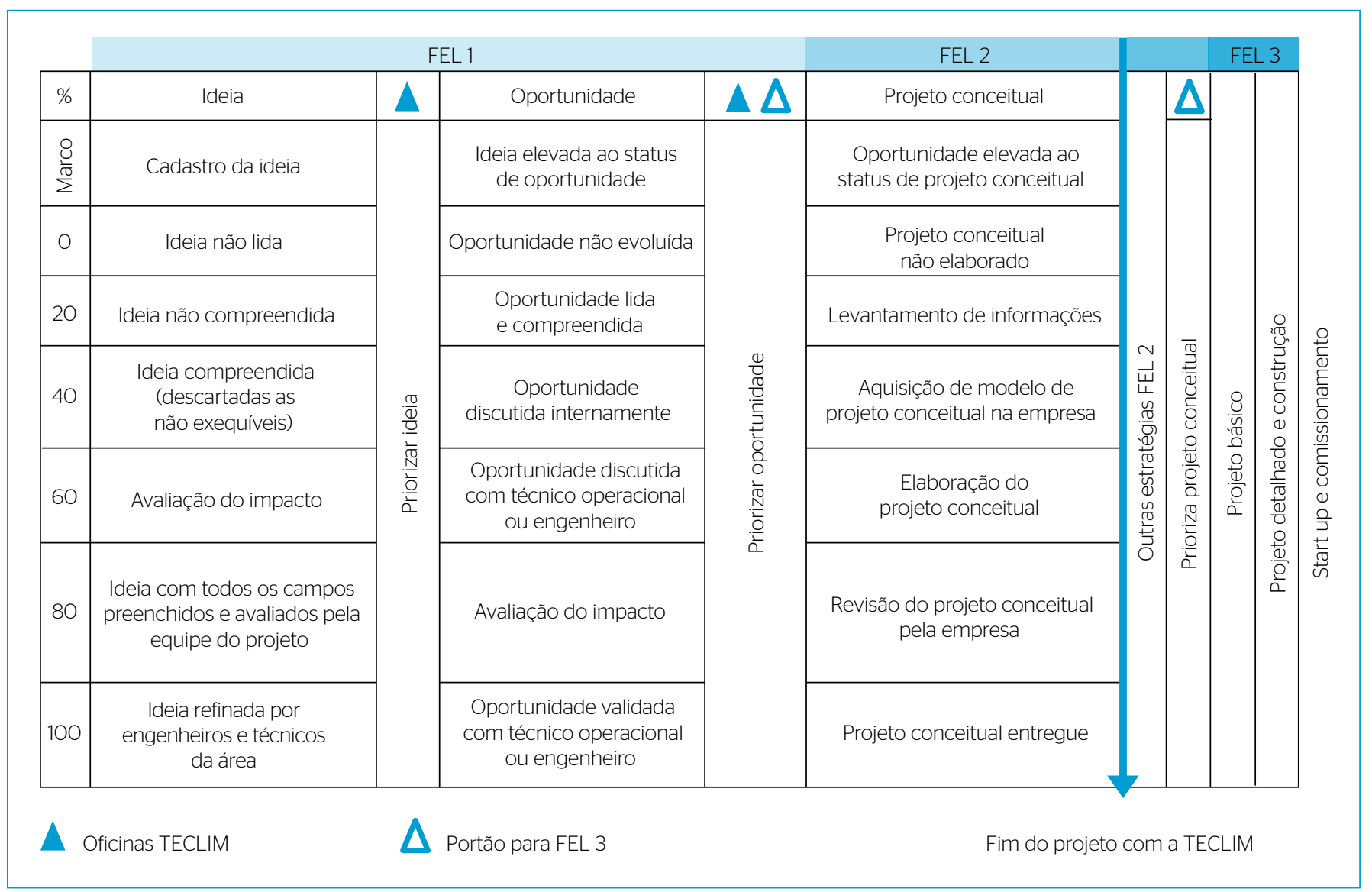

Figura 4 - Sinergia entre o banco de ideias e a metodologia front-end loading com os respectivos detalhamentos em cada fase: ideia, oportunidade e projeto conceitual. 
O percentual de ideias que deu origem aos projetos conceituais, ou seja, que passaram pela aprovação na primeira etapa (FEL 1) e puderam compor o portfólio de projetos (FEL 2) de cada empresa é apresentado na Tabela 3.

Observa-se que o projeto conceitual da TECLIM pode ser completo ou apenas um estudo conceitual com estimativas de investimento e retorno econômico, cultural e ambiental, a depender da disponibilidade de recursos para avaliação das oportunidades. Comparando o percentual de ideias que passaram para a fase FEL 2 nos diversos projetos cooperativos citados na Tabela 3, observa-se um percentual médio de evolução de 15\%, enquanto que pela metodologia FEL a previsão era de $25 \%$. Isso se deve ao tempo requerido no levantamento de informações, na indisponibilidade ou dificuldade de obtenção de valores de medição confiáveis e na carência de mão de obra adequada para essa avaliação, pois com relação à água, as informações disponíveis são escassas e com elevada incerteza.

Constatou-se também que nos projetos A e F, nos quais foram obtidos os melhores resultados de detalhamento, as empresas estiveram mais engajadas na identificação e no estudo de oportunidades, o que caracteriza a importância do comprometimento da empresa.

$\mathrm{Na}$ empresa F, 12\% das ideias passaram direto do FEL 1 para a fase implantação, pois a empresa classificou tais ideias com alta prioridade e optou por implementá-las imediatamente. Esse é um exemplo de que na metodologia do BI uma ideia pode evoluir diretamente para

Tabela 3 - Detalhamento alcançado.

\begin{tabular}{c|c|c|c}
$\begin{array}{c}\text { Projeto } \\
\text { cooperativo }\end{array}$ & Projetos em FEL 1 & Projetos em FEL 2 & FEL1 para FEL2 \\
\hline A & 103 & 20 & $20 \%$ \\
\hline B & 53 & 8 & $15 \%$ \\
\hline C & 113 & 17 & $15 \%$ \\
\hline D & 50 & 5 & $10 \%$ \\
\hline E & 106 & 6 & $6 \%$ \\
\hline F* $^{*}$ & 67 & 16 & $24 \%$ \\
\hline Média & 82 & 12 & $15 \%$ \\
\hline
\end{tabular}

*Na empresa F, 12\% das oportunidades passaram de FEL 1 direto para projeto executivo, sem passar pelo FEL 2 e 3 , pois foram identificadas como de alta prioridade.

Tabela 2 - Ideias geradas e potencial de economia de água e redução de efluentes estimado.

\begin{tabular}{|c|c|c|c|c|c|}
\hline Projeto cooperativo & $\begin{array}{l}\text { Duração } \\
\text { em anos }\end{array}$ & $\mathrm{N}^{0}$ de ideias geradas & Vazão de captação (t.h'1) & $\begin{array}{l}\text { Potencial de economia } \\
\text { de água estimado } \\
\left.\text { (t.h } h^{-1}\right)\end{array}$ & $\begin{array}{l}\text { Potencial de redução } \\
\text { de efluentes estimado } \\
\left.\text { (t. } h^{-1}\right)\end{array}$ \\
\hline A & 2 & 103 & 600 & 158 & 33 \\
\hline B & $6^{\star}$ & 53 & 4300 & 390 & 350 \\
\hline C & 2 & 113 & 407 & 160 & Não estimado \\
\hline D & 2 & 50 & 90 & 32 & 4 \\
\hline F & 1 & 67 & 700 & 300 & 120 \\
\hline \multicolumn{2}{|l|}{ Total } & 492 & 7347 & 1360 & 507 \\
\hline
\end{tabular}

*Ocorreram 3 projetos com duração de 2 anos cada.

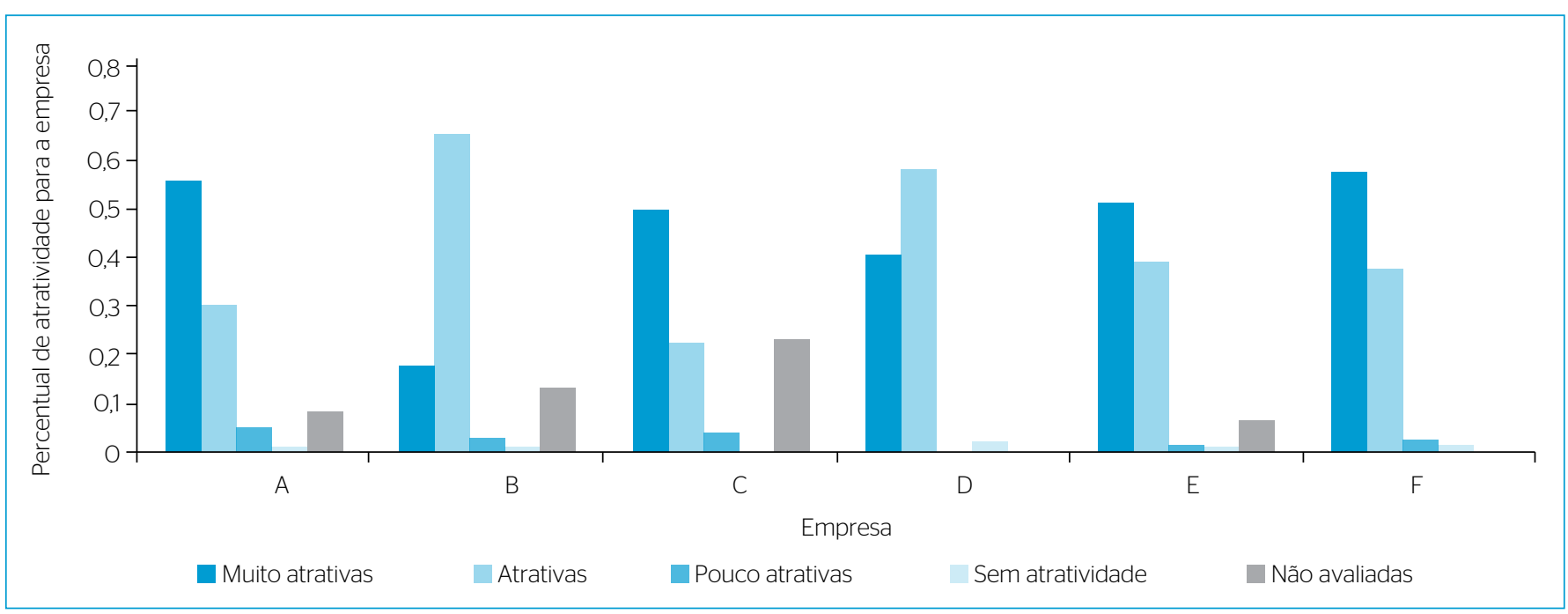

Figura 5 - Resultado da avaliação de atratividade das ideias identificadas nos projetos (fase FEL 1). 
projeto executivo sem passar pelas demais etapas do FEL, conforme demonstrado na Figura 3.

O projeto cooperativo E, por ser o segundo maior em vazões de água captada e um dos mais recentes, foi escolhido para ser o estudo de caso deste artigo.

A participação dos funcionários da empresa no cadastro de ideias é importante para a identificação de ideias alinhadas às necessidades da empresa. A Figura 6 classifica o cadastro de ideias no projeto cooperativo E efetuado pela empresa e pela equipe da TECLIM.

O aumento expressivo no cadastro de ideias pelos funcionários da empresa no mês de janeiro de 2010 (Figura 6), deve-se à realização da oficina para engenheiros e técnicos de operação, na qual foram propostas 35 novas ideias pelos funcionários da empresa. Esse aumento do cadastro de ideias é característico das oficinas de $\mathrm{P}+\mathrm{L}$ (etapa de treinamentos da metodologia TECLIM). Pode-se observar uma participação mais efetiva dos funcionários no BI a partir do mês de agosto de 2009, como resultado da divulgação do projeto na empresa.

Dessa forma, fica mostrado como as etapas de treinamentos e divulgação da metodologia TECLIM influenciam diretamente na utilização e qualidade das ideias cadastradas no BI. A Tabela 4 apresenta o retorno financeiro estimado para a empresa E, com a implementação de parte das ideias cadastradas no BI.

O potencial estimado de economia de água (Tabela 4) equivale a $25 \%$ do consumo de água na empresa E. Esse potencial corresponde apenas a $20 \%$ das ideias geradas, capazes de proporcionar um retorno financeiro de R \$ 5,5 milhões com sua implementação. Logo, as ideias cadastradas no BI demonstram, além de alinhamento estratégico, oportunidade de retorno financeiro para a empresa.
Tabela 4 - Potencial de economia de água e retorno financeiro das ideias do projeto cooperativo $\mathrm{E}$.

\begin{tabular}{|c|c|c|c|}
\hline $\begin{array}{l}\text { No } \\
\text { ideia }\end{array}$ & $\begin{array}{l}\text { Potencial médio de } \\
\text { economia de consumo } \\
\left.\text { de água ( } \mathrm{m}^{3} \mathrm{~h}^{-1}\right)\end{array}$ & $\begin{array}{l}\text { Economia de } \\
\text { água em relação } \\
\text { à captação (\%) }\end{array}$ & $\begin{array}{c}\text { Retorno financeiro } \\
\text { em mil reais } \\
\text { por ano }\end{array}$ \\
\hline 86 & 10,0 & 0,8 & 240 \\
\hline 78 & 3,7 & 0,3 & 70 \\
\hline 74 & VNE & VNE & VNE \\
\hline 72 & VNE & VNE & VNE \\
\hline 71 & VNE & VNE & VNE \\
\hline 64 & VNE & VNE & VNE \\
\hline 52 & 8,2 & 0,7 & 180 \\
\hline 51 & 42,0 & 3,4 & 610 \\
\hline 48 & VNE & VNE & VNE \\
\hline 47 & 27,0 & 2,2 & 490 \\
\hline 44 & 19,6 & 1,6 & 360 \\
\hline 41 & VNE & VNE & VNE \\
\hline 38 & 0,6 & 0,1 & 15 \\
\hline 31 & 9,0 & 0,7 & 160 \\
\hline 18 & 1,7 & 0,1 & 30 \\
\hline 17 & 10,5 & 0,8 & 200 \\
\hline 13 & 45,0 & 3,6 & 800 \\
\hline 11 & 1,2 & 0,1 & 20 \\
\hline 10 & 50,0 & 4,0 & 980 \\
\hline 9 & 30,0 & 2,4 & 440 \\
\hline 6 & 10,0 & 0,8 & 260 \\
\hline 5 & VNE & VNE & VNE \\
\hline 1 & 50,0 & 4,0 & 700 \\
\hline Total & 319,0 & 25,5 & 5500 \\
\hline
\end{tabular}

VNE: valor não estimado

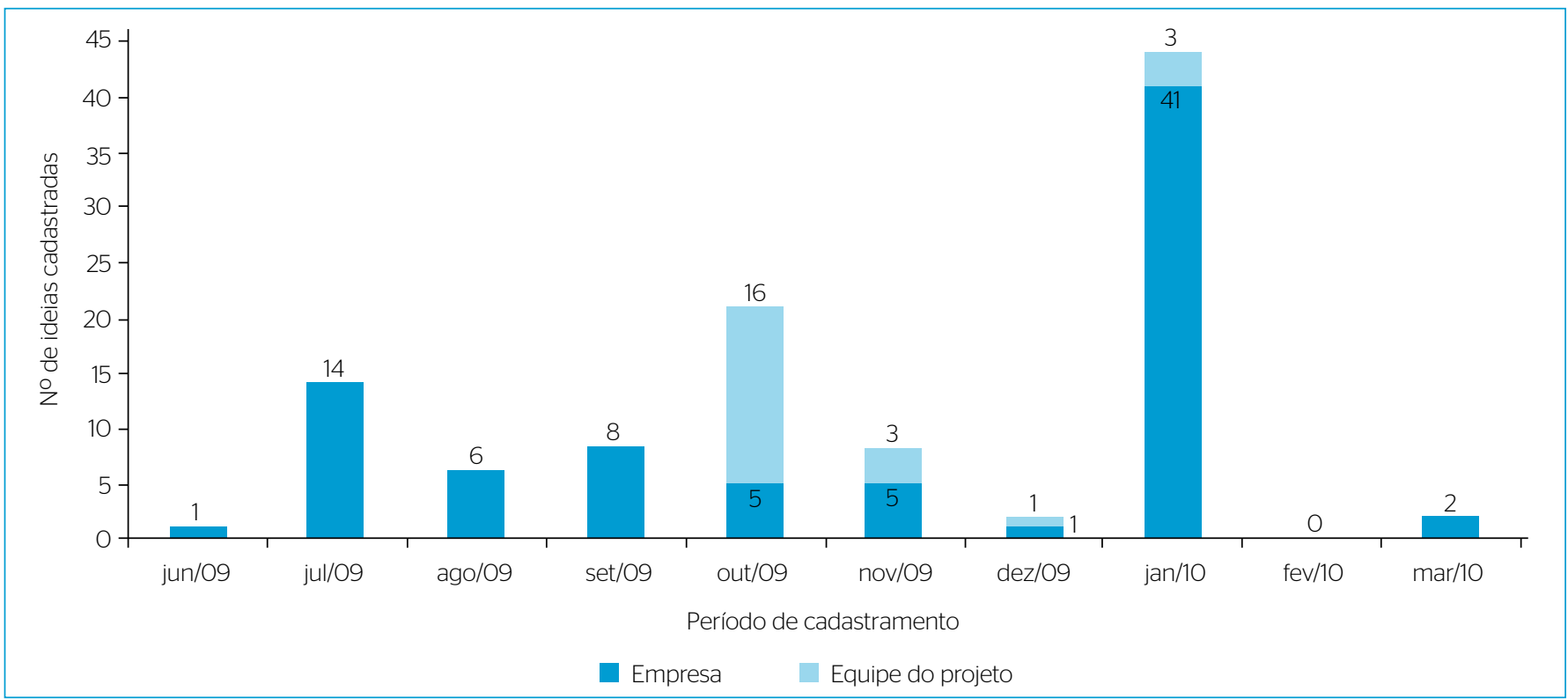

Figura 6 - Contribuições da empresa e da TECLIM no cadastro de ideias ao longo do projeto cooperativo E. 


\section{CONCLUSÕES}

Devido à escassez de recursos e tempo, a ferramenta BI é importante para identificação das oportunidades mais promissoras nos projetos de otimização ambiental da indústria. A utilização de uma ferramenta que explore as fases iniciais do projeto tende a tornar a tomada de decisões mais eficiente, minimizando os custos por mudanças bruscas no escopo, orçamento ou prazos.

As experiências da TECLIM têm mostrado que o percentual de ideias que se consegue detalhar corresponde em média a 15\% do total de ideias cadastradas. Considera-se esse percentual significativo diante do fato de que o uso racional da água pela indústria não costuma ser prioridade nos processos produtivos. Além disso, o grau de incertezas da informação dos fluxos aquosos é elevado, o que torna os estudos mais demandantes de tempo e de recursos.

Por isso a importância do BI, pois otimiza os recursos disponíveis e aumenta a probabilidade de geração de projetos conceituais mais promissores para a empresa e com maior sustentabilidade.

Nos projetos com a indústria, cerca de 500 ideias foram identificadas, representando potenciais estimados de economia de água em torno de $1,4 \times 10^{3} \mathrm{t}^{-1}{ }^{-1}$ o que corresponde ao consumo médio diário de uma cidade com 70 mil residências (considerando domicílios com 4 pessoas e consumo intradomiciliar médio de $120 \mathrm{~L}$ por habitante. dia $^{-1}$, desprezando-se as perdas nos sistemas de abastecimento); e de redução de efluentes em pelo menos $0,5 \times 10^{3}$ t.h h $^{-1}$ O BI auxiliou na definição das oportunidades mais promissoras para a empresa, as quais foram priorizadas e desenvolvidas a projetos conceituais.
Os princípios da metodologia FEL comumente empregados em projetos de grandes empreendimentos puderam ser aproveitados na tomada de decisão da gestão do uso racional da água pela indústria.

Dentre outros resultados, o BI TECLIM em si é um importante produto à medida que funciona como um banco de dados para análise do uso de água pelas indústrias. Uma fonte de informação atualizada continuamente. As informações nele registradas podem ser utilizadas em estudos para traçar perfis de consumo em diversos segmentos industriais, identificar semelhanças de oportunidades inter e entre setores industriais e contribuir para a tomada de decisão do gerenciamento desse recurso pelas empresas.

Outra grande vantagem do BI é a expansão de sua aplicação em outros setores industriais e não industriais, a exemplo de outros projetos desenvolvidos pela TECLIM em prédios públicos, aeroportos e residências. $\mathrm{O}$ uso da ferramenta por empresas, instituições ou pessoas é possível, bastando o interessado cadastrar-se através do sítio www.teclim.ufba.br.

A TECLIM possui um banco de dados raro relacionado ao consumo de água pelas indústrias, que pode ser explorado por pesquisadores para identificação de padrões de consumo e até servir de consultoria entre as empresas. Para a empresa em estudo, além das oportunidades identificadas, o BI pode continuar a ser utilizado para avaliação e detalhamento de oportunidades. O BI, portanto, define-se com uma importante ferramenta de apoio à gestão e à equipe executora de projetos de pesquisa na área de otimização ambiental de processos industriais. Como evolução da ferramenta, pode-se avaliar o percentual de projetos conceituais que foram efetivamente implantados e estão em operação pelas empresas. Uma forma de avaliar a aprovação para a etapa FEL 3 e construir um indicador de eficiência semelhante ao índice FEL para a metodologia TECLIM.

\section{REFERÊNCIAS}

AGÊNCIA NACIONAL DE ÁGUAS - ANA (2009). Conjuntura dos recursos hídricos no Brasil 2009. Brasília: ANA. 204 p.

AGÊNCIA NACIONAL DE ÁGUAS E CONSELHO BRASILEIRO PARA O DESENVOLVIMENTO SUSTENTÁVEL - ANA \& CEBDS. (2006) Fatos e Tendências: água. Brasília: ANA \& CEBDS. 36p. Disponível em: <http://www.ana.gov.br/bibliotecavirtual/arquivos/20070302 094757_\%C3\%81gua,\%2Ofatos\%20e\%2Otend\%C3\%AAncias.pdf>. Acesso em: 07 dez 2010.

ARAYA, A.; CULLICK, S.; SMYTH, J. (2O11) Holist approach for $\mathrm{CO}_{2}$ underground geological storage. Energy Procedia, v. 4, p. 2645-2653.

ÁVILA, S.F. (1995) Controle de processos na geração de efluente orgânico. In: XXXV Congresso Brasileiro de Química, Controle de Processos na Geração de Efluente Orgânico, Anais... Salvador: ABQ. v. 1.

BARSHOP, P. (2004) Best practices pays off. European Chemical News, p. 16-17.
BATAVIA, R. (2001) Front-End Loading for Life Cycle Success. In: Offshore Technology Conference. Houston: OTC.

CARVALHO, E.; KIPERSTOK, A,; KALID, R.A.; ESQUERRE-OLIVEIRA, K.P.; MATTOS, M.C.O. (2009) SIG como ferramenta de gestão do uso industrial de água e geração de efluentes. In: XXV Congresso Brasileiro de Engenharia Sanitária e Ambiental. Recife: ABES.

GOMES, J.F.S.; QUEIROZ, E.M.; PESSOA, F.L.P. (2007) Design procedure for water/wastewater minimization: single contaminant. Journal of Cleaner Production v. 15, n. 5, p. 474-485.

INDEPENDENT PROJECT ANALYSIS - IPA. (2011) Disponível em: <http://www.ipaglobal.com>. Acesso em: O4 out 2011.

JERGEAS, G. (2008) Analysis of the front-end loading of Alberta mega oil sands projects. Project Management Journal, v. 39, n. 4, p. 95-104. 
JERGEAS, G. (2009) Improving construction productivity in Alberta mega oil and gas capital projects. A report submitted to Alberta Finance and Enterprises. Calgary: University of Calgary.

KERKHOF, F.P.J.M. \& VAN BIRGELEN, G. (2011) Greenhouse gas emission reduction anticipating $\mathrm{CO}_{2}$ capture. How ready are you? Energy Procedia, v. 4, p. 2533-2540.

KIPERSTOK, A.; FREIRE, M.T.; KALID, R.A. (2010) Tecnologias poupadoras e água: inovação, aspectos socioculturais e manutenção. In: SOUSA JUNIOR, W.; RIBEIRO, E.N. Uso eficiente da água em aeroportos. Rima, São Carlos.

KIPERSTOK, A.; OLIVEIRA, G.L., OLIVEIRA-ESQUERRE, K.P.; KALID, R.A. (2011) Conservação dos recursos hídricos no semiárido brasileiro frente ao desenvolvimento industrial. In: MEDEIROS, S.S.; GHEYI, H.R.; GALVÃO, C.O.; PAZ, V.P.S. (Eds.). Recursos hídricos em regiões áridas e semiáridas. Campina Grande: Instituto Nacional do Semiárido, cap. 7, p. 207-247.

KIPERSTOK, A.; KALID, R.; SALES, E.A. (2006) Development of water and wastewater minimization tools for the process industry: the experience of the Clean Technology Network of Bahia, Brazil. In: Global Conference on Sustainable Product Development and Life Cycle Engineering, 4 Anais... São Carlos: IFM.

LUCCI, F.A., CONCER, G.M., SANTANA, W.A.F. (2009) A importância de um projeto de pré-detalhamento. Revista Controle e Instrumentação, n. 147.

MARTINS, M.A.F;; AMARO, C.A.; SOUZA, L.S.; KALID, R.A.; KIPERSTOK, A. (2010) New objective function for data reconciliation in water balance from industrial processes. Journal of Cleaner Production, v. 18, p. 1184-1189.

MENEZES, F.C.; OLIVEIRA-ESQUERRE, K.P.; KALID, R.A. (2O11) Predição da dosagem ótima do sulfato de alumínio e do hidróxido de sódio no processo de coagulação da água via redes neurais artificiais. Águas \& Resíduos, v. 14, p. 30-39.

MENDES, C.E.; SOUZA, L.S.; KALID, R.A.; OLIVEIRA-ESQUERRE, K.P.; KIPERSTOK, A. (2011) Assessment of the uncertainty associated with the energy indicator. Renewable and Sustainable Energy Reviews, v. 15, n. 6, p. 3156-3164.

MERROW, E.W.; SONNHALTER, K.A.; SOMANCHI, R.; GRIFFITH, A.F. (2009) Productivity in the UK Engineering Construction Industry. Prepared for Department for Business, Innovation and Skills. Independent Project Analysis, Incorporated. Edited by Paul Gugino. File-BER-0901-SPS.

METCALF \& EDDY, Inc. (2003) Wastewater engineering: treatment and reuse. 3 ed. New York: McGraw-Hill. 1818 p. (McGraw-Hill Series in Civil and Environmental Engineering).

MORAES, F.R.G. (2010) Contribuição ao estudo da concepção de projetos de capital em mega empreendimentos. Dissertação (Mestrado em Construção Civil) - Universidade Federal de Minas Gerais, Belo Horizonte.

MORITA, M.; FLYNN, E.J.; OCHIAI, S. (2O11) Strategic management cycle: the underlying process building aligned linkage among operators practices. International Journal of Production Economics, v. 133, n. 2 , p. $530-540$.
OLIVER, P.; RODRÍGUEZ, R.; UDAQUIOLA, S. (2008) Water use optimization in batch process industries. Part 1: design of the water network. Journal of Cleaner Production, v. 16, n. 12 , p. 1275-1286

OLIVEIRA, G.L.; KALID, R.A.; QUADROS, A.; KIPERSTOK, A.; OLIVEIRA-ESQUERRE, K.; SALES, E. (2009) Manual do Banco de Ideias do Projeto FAFEN-ÁGUA. Documento interno.

OLIVEIRA-ESQUERRE, K.P; KIPERSTOK, A.; KALID, R. A.; SALES, E; TEIXEIRA, L.; PIRES, V.M. (2009) Water and wastewater management in o petrochemical raw material industry. In: 10th International Symposium on Process Engineering. Salvador: PSE.

OLIVEIRA-ESQUERRE, K.P.; KIPERSTOK, A.; MATTOS, M.C.; COHIM, E.; KALID, R.A.; SALES, E.A.; PIRES, V.M. (2O10) Taking advantage of storm and waste water retention basins as part of water use minimization in industrial sites. Resources, Conservation and Recycling, v. 55, n. 3, p. 316-324.

PROJECT MANAGEMENT INSTITUTE - PMI (2004) Um guia do conjunto de conhecimentos em gerenciamentos de projetos. 3a ed. Guia PMBOK, uma norma americana ANSI/ PMI 99-001-2004. Penssylvania: Project Management Institute, Inc.

RUTKOWSKI, E.S. (2010) Front-end loading (FEL) no gerenciamento de projetos. Belo Horizonte: IETEC. Disponível em: <http://www. ietec.com.br/site/techoje/categoria/detalhe artigo/682>. Acessado em: 05 abr 2010.

SANTIAGO, L.P.; CAMPOS E SILVA, D.D.; NOGUEIRA JUNIOR, L.C., MOURA, A.I. (2008) Potencializando o planejamento de projetos: abordagem de uma metodologia de planejamento no contexto do padrão PMBoK. In: XXVIII Encontro Nacional de Engenharia de Produção. Rio de Janeiro: ABEPRO.

SAPANGLER, R.C. (2005) Front end loading and process engineering workflow. Thesis (Master of Science) - The University of Kansas, Kansas.

SAPUTELLI, L.A.; LUJAN, L.; GARIBALDI, L.; SMYTH, J.; UNGREDDA A.; RODRIGUEZ, J.; CULLICK, S. (2008) How integrated field studies help asset team make optimal field development decisions. California: Society of Petroleum Engineers.

UNITED NATIONS ENVIRONMENT PROGRAMME - UNEP. (2O11) Freshwater ecosystems. Disponivel em: <http://www.unep.org/ themes/freshwater/>. Acesso em: 30 dez 2010

VALE. (2007). Relatório de sustentabilidade 2007. Companhia Vale do Rio Doce. Disponível em: < http://www.vale.com/sites/ search/en/Pages/results.aspx?k=relat\%C3\%B3rio\%20de\%20 sustentabilidade\%202007\&u=http://www.vale.com/brasil/EN> Acesso em: 06 out 2011.

WORLD BUSINESS COUNCIL FOR SUSTAINABLE DEVELOPMENT - WBCSD. (2010). Business in the world of water: WBCSD Water Scenarios to 2025. Disponível em: <http://www.wbcsd.org/Pages/ EDocument/EDocumentDetails.aspx?ID=132\&NoSearchContextKe $\mathrm{y}=$ true $>$. Acesso em: 31 dez. 2010

WEIDJE, V.D.G. (2008) Front-end loading in the oil and gas industry. Thesis (Master of Science) - Delf University of Technology, Netherlands. 